\title{
DETECTION OF BACTERIA BASED ON THE
}

\section{THERMOMECHANICAL NOISE OF A}

\section{NANOMECHANICAL RESONATOR: ORIGIN OF THE RESPONSE AND DETECTION LIMITS}

Nanotechnology 19, 035503 (2008) previous manuscript

D. Ramos, J. Tamayo ${ }^{\#}$ J. Mertens, and M. Calleja

BioNanoMechanics Lab, National Centre for Microelectronics, IMM-CNM, CSIC Isaac Newton 8 (PTM), Tres Cantos 28760, Madrid, Spain

L.G. Villanueva

STI-IMM-LMIS1, EPFL - Station 17, CH-1015, Lausanne, Switzerland

A. Zaballos

Genomics Functional Unit, Department of Immunology and Oncology, CNB-CSIC, Darwin 3, Madrid 28049, Spain

We have measured the effect of the bacteria adsorption on the resonant frequency of microcantilevers as a function of the adsorption position and vibration mode. The resonant frequencies were measured from the Brownian fluctuations of the cantilever tip. We found that the sign and amount of the resonant frequency change is determined by the position and extent of the adsorption on the cantilever with regard to the shape of the vibration mode. To explain these results, a theoretical one-dimensional model is proposed. We obtain analytical expressions for the resonant frequency that accurately fits the data obtained by the finite element method. More importantly, the theory data shows a good agreement with the experiments. Our results indicate that there exist two 
opposite mechanisms that can produce a significant resonant frequency shift: the stiffness and the mass of the bacterial cells. Based on the thermomechanical noise, we analyze the regions of the cantilever of lowest and highest sensitivity to the attachment of bacteria. The combination of high vibration modes and the confinement of the adsorption to defined regions of the cantilever allow detection of single bacterial cells by only measuring the Brownian fluctuations. This study can be extended to smaller cantilevers and other biological systems such as proteins and nucleic acids.

PACS numbers: 07.10.Cm, 85.85.+j, 87.15.-v, 05.40.-a

\#Corresponding author: jtamayo@imm.cnm.csic.es 


\section{Introduction}

Progress in nanotechnologies has brought about a number of highly sensitive label-free biosensors. These include electronic biosensors based on nanowires and nanotubes[1,2], optical biosensors based on nanoparticles[3] and mechanical biosensors based on resonat micro- and nanomechanical suspended structures[4]. In these devices, molecular receptors such as antibodies or short DNA molecules are immobilized on the surface of the micro- nanostructures. The operation principle is that molecular recognition between the targeted molecules present in a sample solution and the sensoranchored receptors gives rise a change of the optical, electrical or mechanical properties depending on the class of sensor used. These techniques do not require previous labelling of the sample with fluorescent, colorimetric or radioactive markers. Sample labelling is time consuming, it can interfere with the molecular recognition and also it provides an unspecific background signal. Another fundamental advantage of nanosensors is the inherent small sensing area that allows analysis of very small amounts of sample, a critical issue in many biomedical applications in which the samples or reagents are precious. Moreover, these sensors can be arranged in dense arrays by using established micro- and nanofabrication tools, hence responding to the need for platforms to perform complex chemical and biological analysis.

Nanomechanical resonators are based on the change of the nanometer-scale vibration of a suspended micro- or nanostructure, mostly a cantilever, when molecules adsorb on its surface. This class of sensors is often referred to as resonant mass sensors as the resonant frequency sensitively shifts in proportion to the mass of the molecules adsorbed on the cantilever. The proportionality constant is the effective vibration mass 
of the cantilever. Hence, the detection limits can be greatly enhanced by reducing the size of the resonators. The mass sensitivity has rapidly evolved from the picogram to the attogram range[5,6], by simply reducing the size of the resonators. The vibration of the resonator can be measured with subnanometer resolution by diverse optical and electrical techniques such as the optical beam deflection, optical interferometry, capacitive methods and piezoresistivity.

Nanomechanical resonators have demonstrated that they can detect microorganims with high sensitivity[7-9]. Most of the experiments have been performed in dry air or vacuum where the quality (Q) factor of the resonator is high and therefore the signal-to-noise ratio of the frequency signal is high. However there are applications in which in-situ detection of microbes in aqueous environments is required. This is impeded by the low Q-factor of the resonators as a consequence of the viscosity of the liquids. This difficulty has been overcome by placing the solution inside a hollow resonator that is surrounded by vacuum[10]. In other kind of application, these sensors have been applied for the detection of bacterial growth in humid ambient[11]. It was possible to detect active growth of E. coli cells within one hour, about one order of magnitude shorter than with conventional methods.

In common with many emerging nanotechnology-based sensors, nanomechanical sensors still requires of a major understanding of the mechanisms responsible for the sensor response. This is necessary to obtain i) a correct quantification of the amount of target in the sample and ii) for obtaining those parameters that allow optimization of the sensor response. Recently, we have found that in addition to the added mass, nanomechanical resonators are very sensitive to the 
stiffness of the adsorbed molecules[12,13]. The problem is that both effects produce opposite shifts of the resonant frequency being detrimental to the sensitivity. In the fundamental flexural mode of a singly clamped cantilever, the effect of the adsorbate stiffness can be very important and even dominant in three situations: i) when the adsorption mainly occurs around the clamping region; ii) when the resonators are fabricated in soft materials, such as polymers, with elastic properties similar to that of the adsorbed molecules; and iii) when the cantilevers thickness is comparable to the size of the adsorbed molecules.

Here we have performed an experimental and theoretical study to determine the effect of the adsorption of bacteria Escherichia coli on the eigenfrequencies of a microcantilever. The resonant frequencies are obtained from the Brownian motion, not requiring hence, external excitation. The aim of the study is to ascertain i) how the mechanical properties and mass density of the sample influence on the different eigenfrequencies of the cantilever ii) how both effects can be separated by restricting the adsorption to defined regions of the cantilever and iii) which vibration modes are best suited to obtain high sensitivity based on the thermomechanical noise.

\section{Materials \& methods}

Microdroplets of ultrapure water containing suspended bacteria cells were deposited at different positions along the cantilever by using a home-built inkjet printing set-up. The volume of the droplets were $0.5 \mathrm{nl}$, approximately. The microdispensers were purchased from Microdrop Technologies. The droplet liquid quickly evaporated in few seconds leaving dried clumps of bacteria with a size of 30- 
$100 \mu \mathrm{m}$. The number of deposited cells, between 50 and 4000 cells, was controlled by adjusting the concentration of the bacteria solution and the number of droplets. We used silicon microcantilevers $430 \mu \mathrm{m}$ long, $100 \mu \mathrm{m}$ wide and $1 \mu \mathrm{m}$ thick (Nanoworld). The cantilever motion was measured by the well-known optical beam deflection technique[14].

The cantilever structure is simulated with the finite element software ANSYS. The structure has the same dimensions as the cantilevers used in the experiments. The mesh generated consists of bricks which are $1 \mu \mathrm{m}$ long, $1 \mu \mathrm{m}$ wide and $200 \mathrm{~nm}$ thick.

\section{Experiments}

We measured the resonant peaks of the microcantilevers by means of their inherent Brownian fluctuations[15]. Fig. 1 shows the fast-Fourier transform of the photodetector signal in two orthogonal configurations to discern the flexural and torsional fluctuations. A cross-talk between the flexural and torsional fluctuations is observed. We distinguish the peaks corresponding to the first three flexural modes and first two torsional modes. By fitting the peaks to the harmonic oscillator model we deduce the angular resonant frequencies $(\omega)$ and the quality factors. In a typical experiment these values were: $\omega_{1}{ }^{\mathrm{F}}=2 \pi \cdot 8.5 \mathrm{kHz}, \mathrm{Q}_{1}{ }^{\mathrm{F}}=33, \omega_{2}{ }^{\mathrm{F}}=2 \pi \cdot 51.1 \mathrm{kHz}, \mathrm{Q}_{2}{ }^{\mathrm{F}}=123$ and

$\omega_{3}{ }^{\mathrm{F}}=2 \pi \cdot 139 \mathrm{kHz}, \mathrm{Q}_{3}{ }^{\mathrm{F}}=266 . \omega_{1}{ }^{\mathrm{T}}=2 \pi \cdot 75.6 \mathrm{kHz}, \mathrm{Q}_{1}{ }^{\mathrm{T}}=112, \omega_{2}{ }^{\mathrm{T}}=2 \pi \cdot 229 \mathrm{kHz}, \mathrm{Q}_{2}{ }^{\mathrm{T}}=290$, where the subscript denote the vibration mode and the superscript the kind of vibration, flexural $(\mathrm{F})$ or torsional $(\mathrm{T})$. According to previous studies[16], the results indicate that i) the Q-factor increases with the index of vibration mode and ii) the torsional modes have higher resonance frequencies and Q-factors than their flexural counterparts. For 
the sake of clarity, the study will be restricted to the first three flexural modes and the first torsional mode.

\section{1. Effect of the adsorption position}

The microcantilevers were functionalized with clumps of about $4000 \mathrm{E}$. coli cells by using the ink-jet technique at different positions along the cantilevers. Figure 2 shows the effect of the adsorption position on the resonant frequency of the first three flexural modes and the first torsional mode.

Flexural vs. torsional. Figures 2(a) and 2(b) shows the relative resonant frequency shift of the first flexural and torsional modes as a function of the adsorption position $\left(\mathrm{x}_{0}\right)$. The adsorbed bacteria approximately occupied a region of $100 \times 100 \mu \mathrm{m}^{2}$. Both vibration modes exhibit similar responses. The highest frequency responsivity is achieved when either, the bacterial cells are deposited on near the cantilever free end or near the base. However, the sign of the frequency shift is opposite, being positive for adsorption near the base, and negative for adsorption near the free end. In the flexural mode, this behaviour is related to the added mass and higher flexural stiffness on the cantilever region where the bacteria are deposited. The added mass effect dominates when adsorption is located near the cantilever free end whereas the stiffness effect dominates when adsorption is on near the cantilever fix end. The similar behaviour shown by the torsional mode suggests that the mechanisms responsible for the frequency response are similar as will be demonstrated below. 
Fundamental vs. higher flexural modes. Figures $2 \mathrm{c}$ and $2 \mathrm{~d}$ show the relative resonant frequency shifts of the second and third flexural modes as a function of $\mathrm{x}_{0}$. Except for positions very near the base, the frequency shifts are negative. In addition the relative frequency shifts are smaller than in the case of the fundamental flexural mode. To gain more insight into the nature of the frequency responses the amplitude shapes has been plotted. The relative maxima of absolute frequency shift are found when the bacteria are deposited near the antinodes. The minimal responses are found when the bacteria clumps are near the nodes, where the vibration is small but the bending is maximal. These results suggest that the mass mechanism governs the resonant frequency response in the regions of the beam with higher vibration amplitude. The stiffness contribution would be important for the material adsorbed on the nodes, practically cancelling the mass effect. However, the stiffness effect would not be as important as in the fundamental mode since the bacterial cells occupy an extension relatively large in comparison with the node-antinode distance.

\subsection{Effect of the number of deposited bacterial cells}

Figure 3(a) shows how the resonant frequency of the fundamental flexural (open symbols) and torsional (solid symbols) vibration modes change as a function of the number of adsorbed bacterial cells when the adsorption is located near the clamp region (triangles) and near the free cantilever end (circles). The experimental error was determined by calculating the mean value of the resonant frequency shift as a consequence of the deposition of nanodroplets of ultrapure water without bacterial cells on the cantilever. The error was of about $0.1 \%$ and it is ascribed to the contaminants present in the water and in the ink-jet microdispensers. Our findings were as follows. 
Firstly, the relative resonant frequency shift of the flexural mode is similar to that of the torsional mode. Secondly, independently of the number of cells, the relative resonant frequency shift is positive for both vibration modes when the bacteria clusters are near the clamp, whereas is negative when bacteria are near the cantilever tip. Thirdly, the sensitivity of the resonant frequency shift to the number of bacterial cells is similar for both adsorption positions.

Although the relative resonant frequency shift of the flexural mode and torsional modes are similar, the signal-to-noise ratio clearly enhances with the use of the torsional mode own to the higher Q-factor as shown in Fig. 3(b). In this case, about fifty E. coli cells were deposited near the free cantilever end (see inset pictures in Figs. 3(a) and 3(b)). In general as long as the thermomechanical noise dominates the noise of the system, the use of high vibration modes with higher resonant frequencies and higher Qfactors is advantageous. In our system, the noise from the optical readout limits this practice up to the third flexural mode and second torsional mode (see Fig. 1). We will come back to this point in Sect 5 .

\section{Origin of the resonant response}

\subsection{Theoretical model}

The flexural resonance frequencies can be calculated from the differential EulerBernoulli beam equation modified to take into account the non homogeneous adsorption, 


$$
W\left(\rho_{c} T_{c}+\rho_{a} T_{a}(x)\right) \frac{\partial^{2} u(x, t)}{\partial t^{2}}+\frac{\partial^{2}}{\partial x^{2}}\left(D^{F}(x) \frac{\partial^{2} u(x, t)}{\partial x^{2}}\right)=0
$$

Where $\mathrm{x}$ is the spatial coordinate along the cantilever, $\mathrm{t}$ is the time, $\mathrm{u}$ is the vertical displacement, $\mathrm{W}$ is the cantilever width, $\mathrm{T}$ is the thickness and $\rho$ is the density. Subscripts $\mathbf{c}$ and a denote the cantilever and the adsorbate. $\mathrm{D}^{\mathrm{F}}$ is the cantilever flexural rigidity that relates the bending moment to the change of curvature. The relation between $\mathrm{D}^{\mathrm{F}}$ and the geometry and mechanical properties of the adsorbate and cantilever is given by[17],

$$
D^{F}(x)=\frac{W}{12} \frac{E_{c}{ }^{2} T_{c}{ }^{4}+E_{a}{ }^{2} T_{a}{ }^{4}(x)+2 E_{c} E_{a} T_{c} T_{a}(x)\left(2 T_{c}{ }^{2}+2 T_{a}{ }^{2}(x)+3 T_{c} T_{a}(x)\right)}{E_{c} T_{c}+E_{a} T_{a}(x)}
$$

where $E_{c}$ and $E_{a}$ are the Young's moduli of the cantilever and adsorbed material, respectively.

The differential equation that governs the torsional vibration of a bilayered cantilever can be approximated for a narrow prismatic beam with $\mathrm{W}>>\mathrm{T}_{\mathrm{c}}+\mathrm{T}_{\mathrm{a}}$ and for small twisting angles, $\theta$, as[18],

$$
D^{T}(x) \frac{\partial^{2} \theta(x, t)}{\partial x^{2}}=\left(\rho_{c} J_{c}+\rho_{a} J_{a}(x)\right) \frac{\partial^{2} \theta(x, t)}{\partial t^{2}}
$$

where $\mathrm{J}_{\mathrm{c}}$ and $\mathrm{J}_{\mathrm{a}}$ respectively are the polar moments of inertia of the unloaded cantilever and the adsorbed material given by[19] $\mathrm{J}_{\mathrm{c}}=\left(\mathrm{W}^{3} \mathrm{~T}_{\mathrm{c}}+\mathrm{WT}_{\mathrm{c}}{ }^{3}\right) / 12$ and $\mathrm{J}_{\mathrm{a}}=$ $\left(\mathrm{W}^{3} \mathrm{~T}_{\mathrm{a}}(\mathrm{x})+\mathrm{WT}_{\mathrm{a}}(\mathrm{x})^{3}\right) / 12$; and $\mathrm{D}^{\mathrm{T}}$ is the torsional rigidity. Equation (3) assumes the 
following: i) each cross-section rotates as a rigid body, i.e., the cross-section shape is not distorted; ii) the rate of twist $\partial \theta / \partial \mathrm{x}$ is constant; iii) cross-sections are free to deform along the axis $\mathrm{x}$, being this deformation independent of $\mathrm{x}$. Under these assumptions and by using the membrane analogy introduced by Prandtl[20], we calculate the torsional stiffness,

$$
D^{T}(x)=\frac{W}{3} \frac{G_{c}{ }^{2} T_{c}{ }^{4}+G_{a}{ }^{2} T_{a}{ }^{4}(x)+2 G_{c} G_{a} T_{c} T_{a}(x)\left(2 T_{c}{ }^{2}+2 T_{a}{ }^{2}(x)+3 T_{c} T_{a}(x)\right)}{G_{c} T_{c}+G_{a} T_{a}(x)}
$$

where $G_{c}$ and $G_{a}$ are the shear moduli of the cantilever and adsorbed material, respectively. The shear modulus is related to the Young's modulus by $E=2 G(1+v)$, where $v$ is the Poisson's ratio in each layer. Notice the analogy between the flexural and torsional rigidities.

Eqs. (1) and (3) can not be analytically solved in a general situation. Here, we calculate the resonant frequency of a given vibration mode by performing an energywork balance during a vibration cycle. The accuracy of this method, often referred to as Rayleigh's method[21], depends on how closely one can predict the vibration shape. Here, we assume that adsorption of bacteria on the cantilever does not change the vibration shape of the cantilever. Later on, we will check the validity of this assumption by comparing the analytical results to those obtained by applying the finite element method. 
The torsional vibration can be written as $\theta(\mathrm{x}, \mathrm{t})=\mathrm{A} \Theta_{\mathrm{n}}(\mathrm{x}) \cos \left(\omega_{n}{ }^{T} t+\beta\right)$ where $\Theta_{\mathrm{n}}$ is the shape of the nth-mode torsional vibration of the unloaded cantilever, $\omega_{n}{ }^{T}$ is the nthmode torsional angular eigenfrequency of the loaded cantilever and $\beta$ is an arbitrary phase angle. The mean value of the beam torsion work per oscillation cycle is given by,

$U=\frac{1}{4} \int_{0}^{L} D^{T}(x)\left[\frac{\partial \theta(x, t)}{\partial x}\right]^{2} d x$

Whereas the mean kinetic energy per vibration cycle is given by,

$$
K=\frac{1}{4} \int_{0}^{L}\left(\rho_{c} J_{c}+\rho_{a} J_{a}(x)\right)\left[\frac{\partial \theta(x, t)}{\partial t}\right]^{2} d x
$$

The torsional eigenfrequencies are obtained by equalling the mean potential and kinetic energies per oscillation cycle,

$$
\omega_{n}^{T}=\left(\frac{\int_{0}^{L} D^{T}(x)\left[\frac{d^{2} \Theta_{n}(x)}{d x^{2}}\right]^{2} d x}{\rho_{c} J_{c} \int_{0}^{L}\left[1+\frac{\rho_{a} J_{a}(x)}{\rho_{c} J_{c}} \Theta_{n}^{2}(x) d x\right.}\right)^{1 / 2}
$$

The eigenfrequencies of the flexural modes can similarly be obtained[12,13], 


$$
\omega_{n}^{F}=\left(\frac{\int_{0}^{L} D^{F}(x)\left(\frac{d^{2} \Psi_{n}(x)}{d x^{2}}\right)^{2} d x}{\rho_{c} W T_{c} \int_{0}^{L}\left(1+\frac{\rho_{a}}{\rho_{c}} \frac{T_{a}(x)}{T_{c}}\right) \Psi_{n}^{2}(x) d x}\right)^{1 / 2}
$$

The torsional and flexural vibration mode shapes for the unloaded cantilever are given by,

$\Theta_{n}(x)=\tan \left(k_{n}^{T} L\right) \sin \left(k_{n}^{T} x\right)+\cos \left(k_{n}^{T} x\right)$

$\Psi_{n}(x)=\sin k_{n}^{F} x-\sinh k_{n}^{F} x+\frac{\left(\sin k_{n}^{F} L+\sinh k_{n}^{F} L\right)\left(\cosh k_{n}^{F} x-\cos k_{n}^{F} x\right)}{\cos k_{n}^{F} L+\cosh k_{n}^{F} L}$

where $\Theta_{0}$ and $A_{n}$ are respectively the arbitrary values of the oscillation amplitude for the torsional and flexural vibrations. The flexural eigenvalues satisfy the equations $1+\cos \left(k_{n}{ }^{F} L\right) \cosh \left(k_{n}{ }^{F} L\right)=0$ and $\cos \left(k_{n}{ }^{T} L\right)=0$, which give $k_{n}{ }^{F}=1.8751,4.6941,7.8548, \ldots$ Whereas the torsional eigenvalues are given by the poles of the boundary condition $\theta(\mathrm{x}=\mathrm{L})=\Theta_{0} / \cos \mathrm{k}_{\mathrm{n}}{ }^{\mathrm{T}} \mathrm{L}$.

\subsection{Comparison between theory, finite element simulations and experiments}

We calculate the resonant frequency shifts of several vibration modes induced by the adsorption of bacterial cells on a localized region of the cantilever. The bacteria clumps on the cantilever are modelled as a continuum solid square pad. In order to compare with the experiments, we assume that the pad contains 4000 cells and it has a lateral size of $100 \mu \mathrm{m}$. By equalling the volume of the all bacterial cells to the volume of a pad, we deduce an effective thickness of $800 \mathrm{~nm}$, smaller than the bacteria 
thickness, $1 \mu \mathrm{m}$. We set the Young's modulus of the biolayer to $1 \mathrm{GPa}$, which is in the range of values obtained by atomic force microscopy measurements in dried bacteria[22]. The calculations are performed by applying the one-dimensional analytical approach developed in Sect. 4, Eqs. (7) and (8). A summary of the results is shown in Fig. 3 for the first three flexural modes and the first torsional mode.

Fig. 4 shows the theoretical results of the relative resonant frequency shift of the first three flexural modes and first torsional mode as a function of the adsorption position (lines). The theoretical results shown in Fig. 4 exhibit all the relevant features observed in the experimental data. These features can be explained by close inspection of equations (7) and (8). Adsorption of bacteria brings about two effects; i) a local increase of the mass per unit length of the beam that induces a decrease of the resonant frequency; and ii) a local increase of the cantilever flexural rigidity that induces an increase of the resonant frequency. The proportionality factors for these effects are the amplitude and curvature of the vibration shape, respectively. For both the first flexural and torsional modes, the regions of highest amplitude and highest curvature are located near the free and fixed ends of the cantilever, respectively. Thus, despite the quantitative differences in the vibration shapes of the first flexural and torsional modes, the resonant frequency shifts of both are positive and dominated by the stiffness of the bacterial cells when they adsorb on near the clamp; whereas the frequency shifts are negative and dominated by the mass loading when the bacteria are near the free cantilever end. Following the same reasoning, the local maxima and minima of the resonant frequency shifts for the second and third flexural modes are related to the adsorption on regions around the vibration nodes (regions of null vibration and high curvature) and regions around the antinodes (relative maxima of amplitude). The quantitative discrepancy 
between the theory and the experiments is mainly related to the differences in the number of bacteria between different experiments. This difference can be of about $30 \%$. In addition, uncertainties in the values of the Young's modulus and density of the bacteria as well as the theoretical simplification of the bacteria clumps as continuum homogeneous pads can produce significant quantitative differences. However, despite the simplicity of our model, the experiments are well-described by the theory.

To check the accuracy of our theoretical model we have also performed finite element method (FEM) simulations. In fig. 4, the theoretical results (lines) and the FEM data (symbols) are shown. For the flexural modes, the theoretical results perfectly match the data obtained by FEM (symbols). However, a discrepancy is found for the torsional mode in a wide region around the cantilever fixed end. This deviation is mainly related to the differential equation applied to describe the torsional vibration of a beam, eq. (3). Basically, the accuracy of our model to predict the resonant frequencies depends on how closely the expressions for the shape of the vibration modes (Eqs. (9a) and (9b)) follow the real vibration. We have found that the Euler-Bernoulli differential equation provides vibration shapes very accurate, with a mean deviation with respect to the FEM simulations below $0.6 \%$. Moreover, the FEM simulations indicate that the bacteria adsorption has a negligible influence on the vibration shapes. However, the differential equation used to model the torsional vibration provides a vibration shape that differs $5.3 \%$ with respect to the vibration shape obtained by FEM simulations. This discrepancy is related to the assumptions about the cross-sectional deformation of the beam that must be taken to obtain an analytical differential equation. 


\section{Thermomechanical limit}

The optimization of the sensitivity of nanomechanical resonators for sensing biological adsorption requires i) minimizing the frequency noise and ii) maximizing either, the stiffness or the mass effect on the resonant frequency, whereas minimizing the other effect. The last requirement can be obtained by limiting the adsorption to functionalized regions of the cantilever whose position and size depend both on the used vibration mode. The choice of the mass or stiffness of the sample as the parameter measured by the sensor will depend on the relative density, thickness and Young's modulus of the adsorbed material with respect to those of the cantilever.

\subsection{Frequency noise}

Let us assume that the noise of the system is limited by the thermomechanical noise, and that the only source of excitation is the random thermal force originated from the surrounding molecules colliding the cantilever[23,24]. In this situation, the spectral density of the cantilever Brownian motion is given by $\mathrm{S}_{\mathrm{th}}(\omega)=|\mathrm{X}(\omega)|^{2} \psi_{\text {th }}$, where $\mathrm{X}(\omega)$ is the mechanical susceptibility of the resonator and $\psi_{\text {th }}$ is the spectral density of the random Langevin force. The spectral density $\psi_{\text {th }}$ can be written as $\psi_{\mathrm{th}}=4 \gamma \mathrm{k}_{\mathrm{B}} \mathrm{T}$ where $\mathrm{k}_{\mathrm{B}}$ is the Boltzmann's constant, $\mathrm{T}$ is the absolute temperature and $\gamma$ is the damping constant. The mechanical susceptibility can be written as,

$$
X_{\text {eff }}(\omega)=\sum_{n}\left\{m_{n}\left(\omega_{n}^{2}-\omega^{2}\right)+i \gamma_{n} \omega\right\}^{-1}
$$


where the subscript $n$ indexes the vibration mode and $m_{n}$ is the mode-dependent effective mass. The Q-factor of the $\mathrm{n}^{\text {th }}$ vibration mode is given by $\mathrm{Q}_{\mathrm{n}}=\mathrm{m}_{\mathrm{n}} \omega_{\mathrm{n}} / \gamma_{\mathrm{n}}$. Let us consider a time interval $\tau$ long, in which the output signal $p(t)$ is bandpass filtered around the resonant frequency $\omega_{\mathrm{n}}$. The output signal can then, be described as a sine wave of frequency $\omega_{\mathrm{n}}$ with slowly varying and randomly modulated phase, $\phi(\mathrm{t})$ and amplitude $r(t)$, i.e., as $p(t)=r(t) \cos \left[\omega_{n} t+\phi(t)\right]$. By applying Fourier series we can write the amplitude and phase as $\mathrm{r}^{2}(\mathrm{t})=\mathrm{x}^{2}(\mathrm{t})+\mathrm{y}^{2}(\mathrm{t})$ and $\tan \phi(\mathrm{t})=\mathrm{y}(\mathrm{t}) / \mathrm{x}(\mathrm{t})$ where,

$$
\begin{aligned}
& x(t)=\sum_{k} \sqrt{\left|H\left(k \frac{2 \pi}{\tau}\right)\right|^{2} S_{t h}\left(k \frac{2 \pi}{\tau}\right) \frac{2}{\tau}} \cos \left[\left(k \frac{2 \pi}{\tau}-\omega_{n}\right) t+\alpha_{k}\right] \\
& y(t)=\sum_{k} \sqrt{\left.H\left(k \frac{2 \pi}{\tau}\right)\right|^{2} S_{t h}\left(k \frac{2 \pi}{\tau}\right) \frac{2}{\tau}} \sin \left[\left(k \frac{2 \pi}{\tau}-\omega_{n}\right) t+\alpha_{k}\right]
\end{aligned}
$$

where $H(\omega)$ is the transfer function of the bandpass filter that is 1 if $\omega_{n}-B<\omega<\omega_{n}+B$ and zero for the other frequencies, and $\alpha_{\mathrm{k}}$ are uniformly distributed random phase angles that ensures that $\mathrm{p}(\mathrm{t})$ is Gaussian-distributed. The phase fluctuations can be viewed as frequency fluctuations by applying the relation $\delta \omega_{n}(t) \equiv \omega(t)-\omega_{n}=d \phi / d t$, where $\omega(t)$ is the instantaneous frequency. For sufficiently long intervals, $2 \pi / \tau<<\omega_{0}$, the ensemble-mean square frequency fluctuation is given by,

$$
\left\langle\delta \omega_{n}(t)^{2}\right\rangle=\frac{\pi}{\omega_{0} \tau} \frac{\int_{\omega_{n}-B}^{\omega_{0}+B} S_{t h}(\omega)\left(\omega-\omega_{n}\right)^{2} d \omega}{\int_{\omega_{n}-B}^{\omega_{n}+B} S_{t h}(\omega) d \omega}
$$


For the practical case in which $\omega_{\mathrm{n}} / \mathrm{Q}_{\mathrm{n}}<<2 \pi / \tau<<\omega_{\mathrm{n}}$ the frequency noise equation reduces to the simple expression $\left\langle\delta \omega_{\mathrm{n}}(\mathrm{t})^{2}\right\rangle \cong \mathrm{B} /\left(\tau \mathrm{Q}_{\mathrm{n}}\right)$. In this case, the noise primarily depends on the Q-factor. Thus, the noise can be significantly reduced by using vibration modes with high Q-factors. For instance, this is illustrated in Fig. 3(b), in which the frequency shift due to the bacteria attachment can be hardly appreciated in the flexural mode, whereas it is clearly observed in the torsional mode.

\subsection{Detection limits}

Substituting eqs. (7) and (8) into eq. (12), we plot the signal-to-noise ratio (S/N) of the absolute resonant frequency shift induced by the biological layer used in the theory to model the bacteria cells, as a function of the longitudinal size and centre position of adsorption (Fig. (5)). The adsorbate size and adsorption position are normalized to the length of the cantilever $(430 \mu \mathrm{m})$ to ease the application of the results to smaller cantilevers and adsorbates. The acquisition time is set to $2.5 \mathrm{~s}$ and $\mathrm{B} / \omega_{\mathrm{n}}$ to 10 $\%$, in order to simulate our experiments. The resulting three-dimensional plot allows to asses the regions of the cantilever and vibration modes more sensitive to adsorption of bacteria. The black regions are those in which $\mathrm{S} / \mathrm{N}$ is smaller than one, and therefore correspond to regions in which the adsorption of bacteria can not be detected. The sign of the resonant frequency shift is marked with plus and minus symbols. The first flexural and torsional modes are insensitive to bacteria adsorption when the adsorption position is of about 180 and $160 \mu \mathrm{m}$ respectively. In both modes, the most sensitive adsorption region is near the free cantilever end where the resonant frequency shift is dominated by the added mass. The torsional mode has a higher $\mathrm{S} / \mathrm{N}$ due to the higher $\mathrm{Q}$ and higher resonant frequency. The second and third flexural modes have higher $\mathrm{S} / \mathrm{N}$ 
and the number of blind regions increase to three and four respectively. However the blind regions are narrower than in the flexural and torsional modes. The resonant frequency shifts are negative except in the region near the clamping. The most sensitive region for both modes is near the free end where the resonance response is dominated by the mass effect. However the clamp region is also very sensitive and can be used for bacteria detection based on the frequency shift caused by the local increase of stiffness.

A summary of the results is shown in table I, in which we show the minimum number of bacteria cells that can be detected when the bacteria are positioned on the clamping or on the free end. The attached bacteria are supposed to be randomly oriented in the cantilever plane. The results show that the third flexural mode is the most sensitive and it can detect between 2 cells on the free cantilever end and about 6 cells near the clamping region. This mode is of about 18 times more sensitive than the

fundamental flexural mode. The results indicate that the sensitivity can be enhanced by the use of higher vibration modes. However, when the size of the adsorbate approaches to the free end/node or fixed end/antinodes distances the use of high vibration modes is not advantageous. In these cases, the opposite effects of the mass and stiffness of the adsorbate degrades the sensitivity of the nanomechanical resonator.

\section{Conclusions}

We demonstrate that adsorption of bacteria on a resonant cantilever can produce either a negative or positive resonant frequency shift. The sign and amount of the resonant frequency shift depend both on the position and extent of the bacterial cells on the cantilever with regard to the shape of the vibration mode used in the measurement. This 
behaviour is due to two effects: i) the stiffness of the bacterial cells that increase the cantilever flexural rigidity giving rise a positive contribution to the resonant frequency and ii) the well-known added mass effect that gives rise a negative shift of the resonant frequency. The resonant response is dominated by the mass when the bacteria adsorb on regions of high vibration amplitude as on the antinodes and the free cantilever end. The stiffness effect dominates when the adsorption is on regions of small vibrations as the nodes and the clamp region. The optimization of the sensitivity of nanomechanical resonators to biological adsorption requires i) minimizing the frequency noise and ii) maximizing either, the stiffness or the mass effect on the resonant frequency, whereas minimizing the other effect. Based on the analysis of the thermomechanical noise, we conclude that, in this case, the use of higher vibration modes with sensitised regions near the free end or fixed end is the best strategy to enhance the sensitivity. The size of the optimal sensing region decreases with the index of the vibration mode used, setting and uppermost limit that will depend on the size of the targeted biological sample. This study can be applied for smaller cantilevers and other biological systems such as virus, proteins or nucleic acids.

D.R. acknowledges the fellowship funded by the Autonomic Community of Madrid. The authors acknowledge A. Zaballos for supplying the bacteria cells. This research was supported by Spanish Ministry of Science under grant No. TEC2006-10316. 


\section{References}

[1] Rosi N L and Mirkin C A 2005 Chem. Rev. 1051547.

[2] Stern E, Klemic J F, Routenberg D A, Wyrembak P N, Turner-Evans D B, Hamilton

A D, LaVan D A, Fahmy T M and Reed M A 2007 Nature 445519.

[3] Taton T A, Mirkin C A and Letsinger R L, 2000 Science 2891757.

[4] Bietsch A, Zhang J, Hegner M, Lang H P and Gerber C, 2004 Nanotechnology 15 873.

[5]Illic B, Craighead H G, Krylov S, Senaratne W, Ober C and Neuzil P. 2004 J. Appl. Phys. 953694.

[6]Ekinci K L, Yang Y T and Roukes M L 2004 J. Appl. Phys. 952682.

[7]Illic B, Czaplewski D, Craighead H G, Neuzil P, Campagnolo C and Batt C 2000 Appl. Phys. Lett. 77450.

[8]Gupta A, Akin D and Bashir R 2004 Appl. Phys. Lett. 841976.

[9]Gupta A K, Nair P R, Akin D, Ladisch M R, Broyles S, Alam M A and Bashir R 2006 PNAS 10313362.

[10]Burg T P, Godin M, Knudsen S M, Shen W, Carlson G, Foster J S, Babock K, Manalis S R 2007 Nature 4461066.

[11]Gfeller K Y, Nugaeva N and Hegner M, 2005 Biosensors and Bioelectronics 21 528.

[12]Ramos D, Tamayo J, Mertens J, Calleja M and Zaballos A 2006 J. Appl. Phys. 100 106105.

[13]Tamayo J, Ramos D, Mertens J and Calleja M 2006 Appl. Phys. Lett. 89224104. 
[14]Putman C A J, De Grooth B G, Van Hulst N F and Greve J 1992 J. Appl. Phys. 72 6.

[15]Schäffer T E 2005 Nanotechnology 16664.

[16]Chon J W M, Mulvaney P, Sader J E 2000 J. Appl. Phys. 873978.

[17]Sandberg R, Svendsen W, Molhave K, and Boisen A, 2005 J. Micromech.

Microeng. 151454.

[18]Reinstädtler M, Kasi T, Rabe U, Bhushan B and Arnold W 2005 J. Phys. D: Appl. Phys. 38269.

[19]Song Y and Bhushan B 2006 Microsyst. Technol. 12219.

[20]Den Hartog J P, 1952 “Advanced Strength of Materials”, McGraw-Hill, New York [21]Stephen N G 1989 J. sound. Vib. 131345

[22]Yao X, Jericho M, Pink D and Beveridge T 1999 J. Bacteriol. 1816865.

[23]Dürig U, Züger O and Stalder A, 1992 J. Appl. Phys. 721778.

[24]Saulson P R 1990 Phys. Rev. D 422437. 


\section{TABLES}

Table I. Minimum number of bacteria cells that can be detected as a function of the vibration mode and the adsorption position.

\begin{tabular}{|l|l|l|l|l|}
\hline & $1^{\text {st }}$ Flexural & $1^{\text {st }}$ Torsional & $2^{\text {nd }}$ Flexural & $3^{\text {rd }}$ Flexural \\
\hline Clamp & 81 & 75 & 15 & 6 \\
\hline Free end & 36 & 33 & 7 & 2 \\
\hline
\end{tabular}




\section{FIGURE CAPTIONS}

Fig. 1. Frequency spectra of the Brownian fluctuations of the cantilever (430 $\mu \mathrm{m}$ long, $100 \mu \mathrm{m}$ wide and $1 \mu \mathrm{m}$ thick) for two transversal configurations of the quadrant photodiode showing the torsional (dashed line) and flexural (continuous line) resonant peaks.

Fig. 2. Experimental measurements of the relative resonant frequency shift as a function of the longitudinal position of the adsorbed bacteria with respect to the clamping for the first three flexural and first torsional modes. The dashed lines are polynomial interpolations for eye-guide. The solid lines are the vibration shape for the second and third flexural modes.

Fig. 3. (a) Relative frequency shift of the fundamental flexural (open symbols) and torsional (solid symbols) vibration modes as a function of the number of adsorbed $E$. coli cells. The bacteria were deposited near the cantilever base (triangles) and near the cantilever free end (circles). The dashed region represents the experimental error due to the non-specific adsorption of material during the deposition of bacteria. The inset shows an optical picture of a cantilever with approximately fifty bacteria on the tip. (b). Frequency spectra of the fundamental flexural (top) and torsional (bottom) modes of the cantilever with approximately 50 bacteria near the tip (inset in (a)). Inset shows a zoom of the inset in (a). 
Fig. 4. Theoretical calculations of the relative resonant frequency shift as a function of the longitudinal position $\left(\mathrm{x}_{0}\right)$ of the adsorbed bacteria with respect to the clamping for the first three flexural and first torsional modes (lines). The graph also includes the data obtained by using the finite element method (symbols). A schematic cartoon of the cantilever and adsorbate is also shown.

Fig. 5. Signal-to-noise ratio $(\mathrm{S} / \mathrm{N})$ of the absolute resonant frequency shift induced by the biological layer used to model the bacteria cells, as a function of the longitudinal size and centre position of adsorption. The black regions are those in which $\mathrm{S} / \mathrm{N}$ is smaller than one, and therefore correspond to regions in which the adsorption of bacteria can not be detected. The sign of the resonant frequency shift is marked with plus and minus symbols. 
FIGURE 1

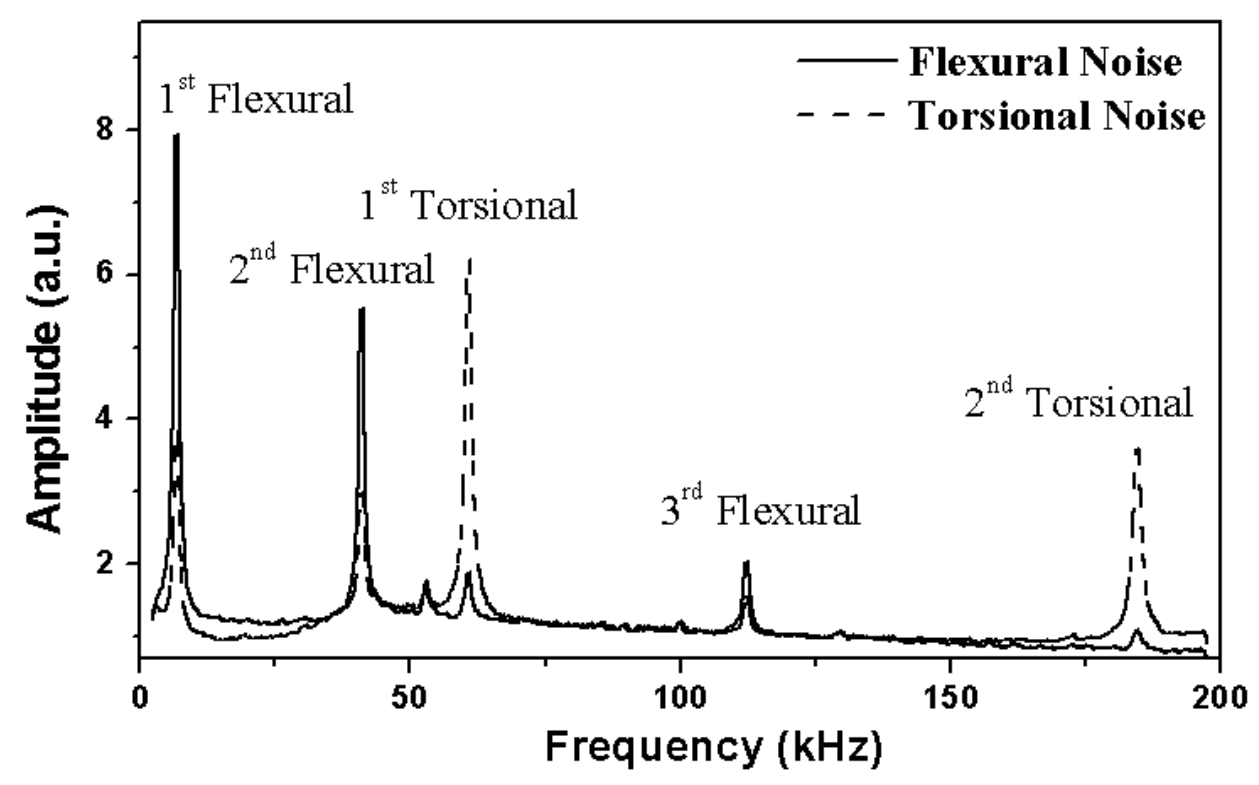


FIGURE 2

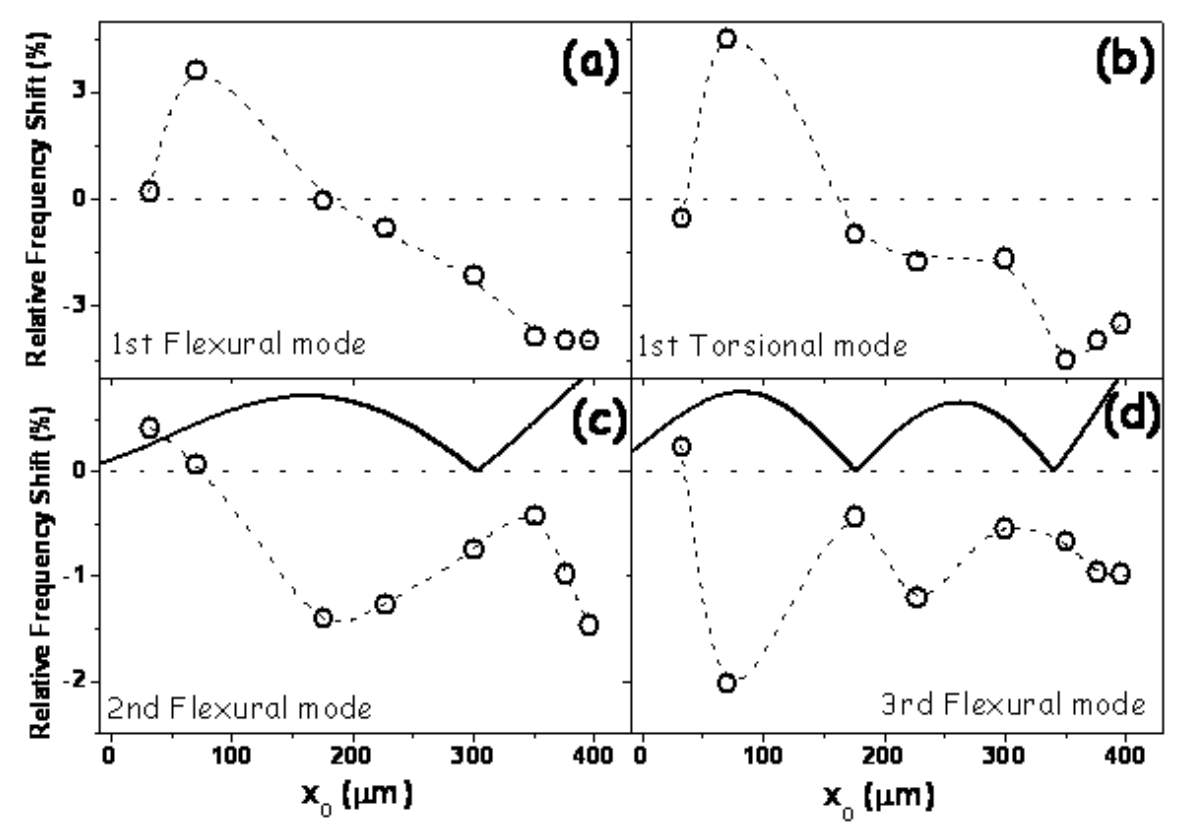


FIGURE 3

(a)

(b)

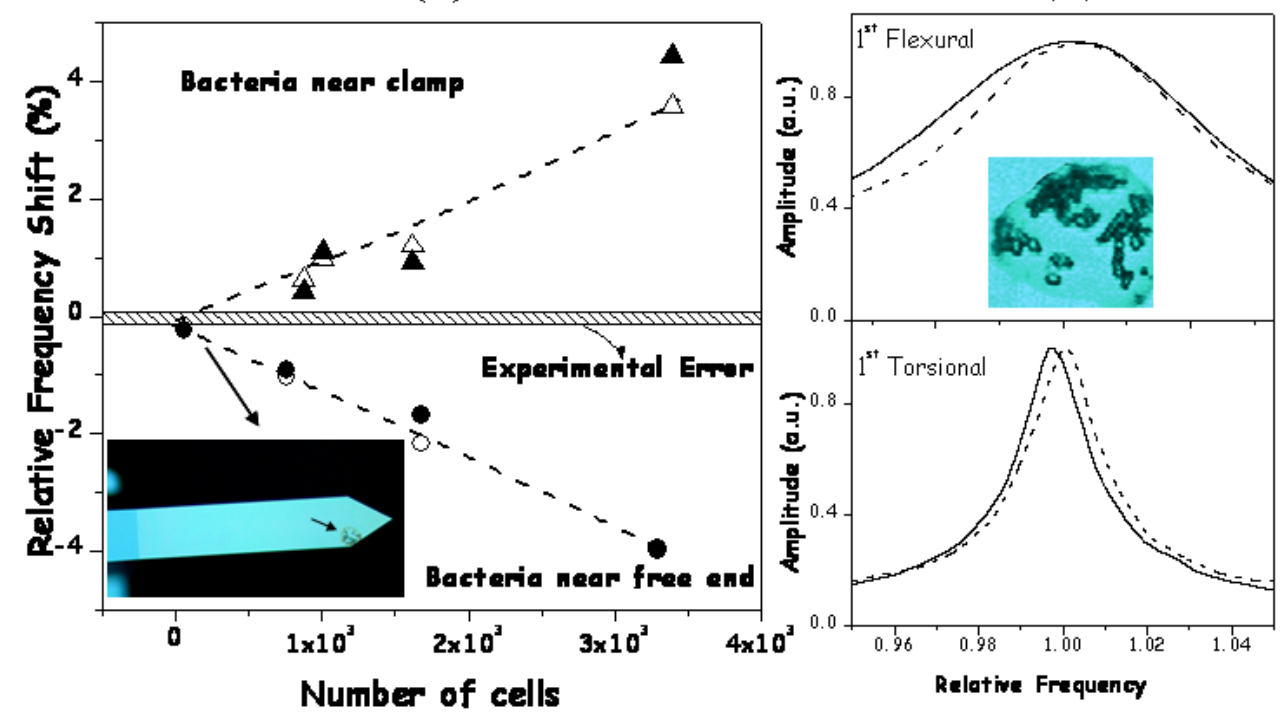


FIGURE 4

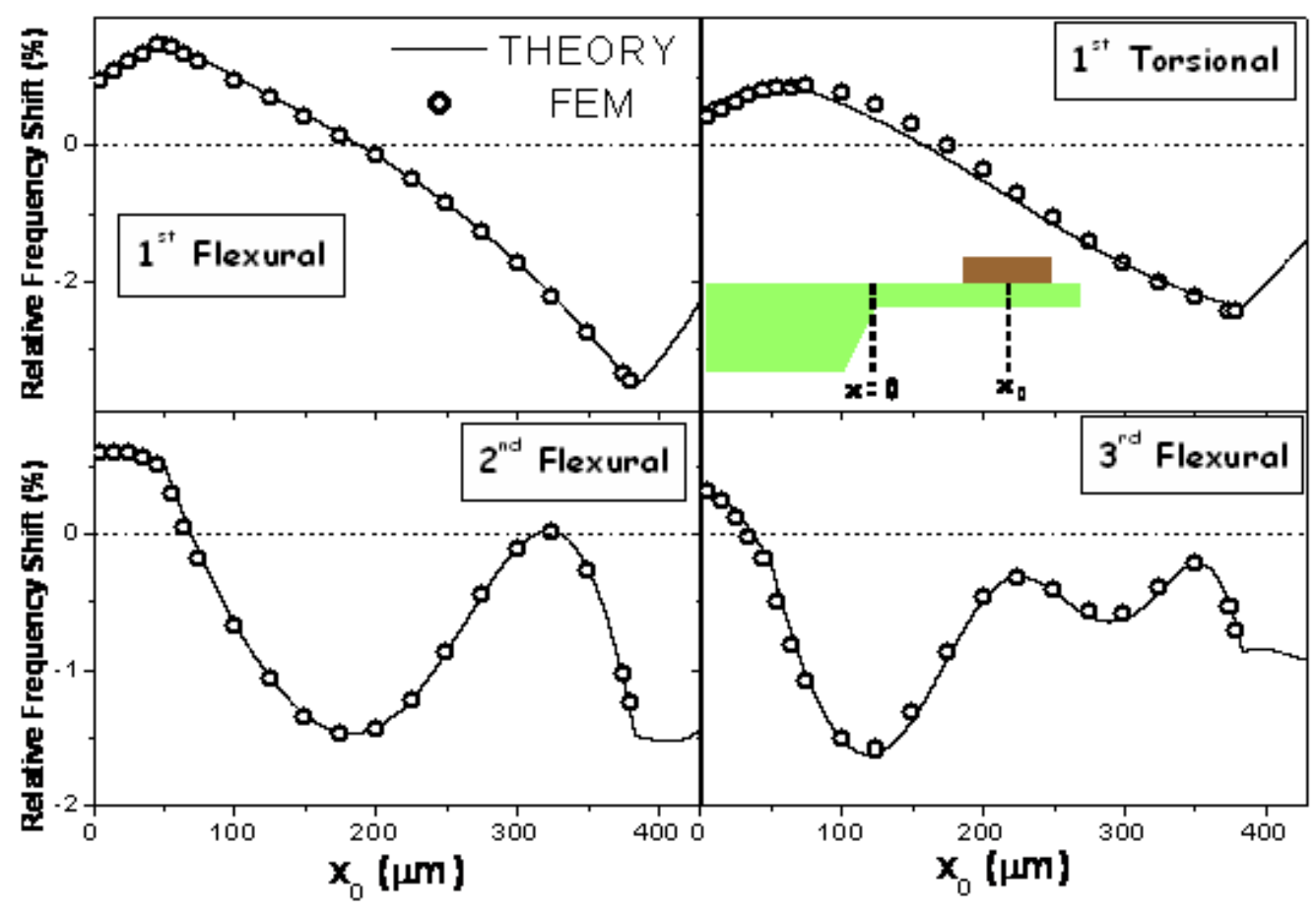




\section{FIGURE 5}

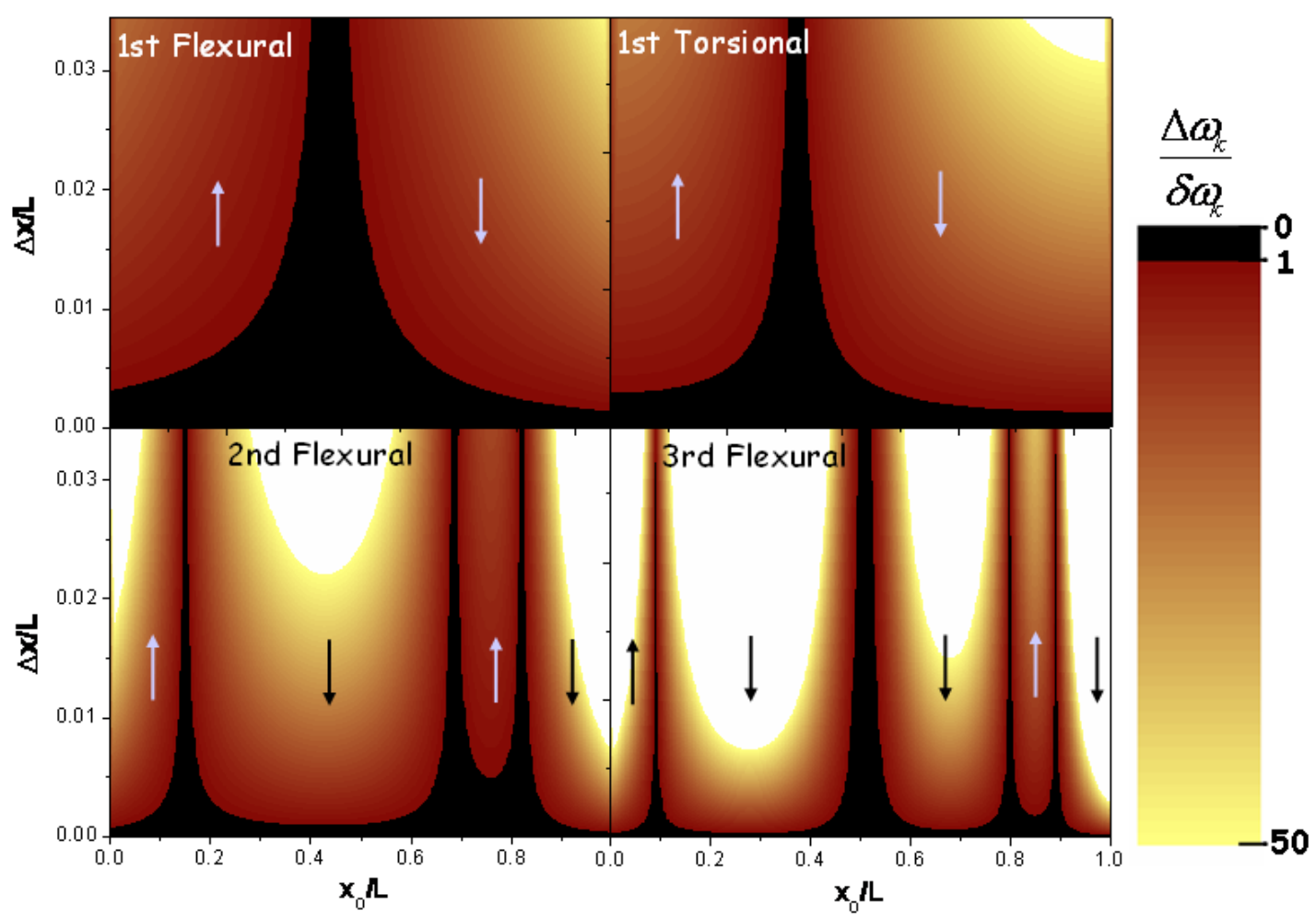

